\title{
Limitation of high-resolution melting curve analysis for genotyping simple sequence repeats in sheep
}

\author{
M. Yang',2*, Y.J. Yue ${ }^{1 *}$, T.T. Guo ${ }^{1}$, J.L. Han ${ }^{1}$, J.B. Liu' ${ }^{1}$, J. Guo ${ }^{1}$, X.P. Sun ${ }^{1}$, \\ R.L. Feng ${ }^{1}$, Y.Y. Wu ${ }^{1,2}$, C.F. Wang ${ }^{1,2}$, L.P. Wang ${ }^{1}$ and B.H. Yang ${ }^{1}$ \\ ${ }^{1}$ Lanzhou Institute of Husbandry and Pharmaceutical Sciences of Chinese \\ Academy of Agricultural Sciences, Lanzhou, China \\ ${ }^{2}$ College of Animal Science and Technology, Gansu Agricultural University, \\ Lanzhou, China \\ *These authors contributed equally to this study. \\ Corresponding author: B.H. Yang \\ E-mail: yangmindk@163.com / yangbh2004@163.com
}

Genet. Mol. Res. 13 (2): 2645-2653 (2014)

Received June 21, 2013

Accepted October 25, 2013

Published April 8, 2014

DOI http://dx.doi.org/10.4238/2014.April.8.7

\begin{abstract}
Variation in microsatellite or simple sequence repeat (SSR) loci has, until recently, relied heavily on the use of gel-based methods that can be both time consuming and difficult to genotype. Non gel-based systems are therefore important to increase simplicity and improve turn-around time without compromising assay sensitivity and accuracy. In this report, we assessed the latest of the non-gelbased methods, high-resolution melting (HRM) curve analysis. HRM is a technique that monitors exactly the decreasing fluorescence of intercalating dye in the process of dissociation of double-stranded DNA. The measurement immediately follows polymerase chain reaction in a one-step, closed-tube method. Four SSR loci of different complexity in sheep, namely MAF209, MCM140, CB226, and SRCRSP5, were assessed using the LightScanners System with LC Greens PLUS DNA binding dye. In order to improve the accuracy of genotyping,
\end{abstract}


we applied internal oligo nucleotide calibrators while performing HRM. DNA polymorphisms were previously identified using capillary electrophoresis analysis (CE). The result showed that CE detected more genotypes than HRM in the same loci regardless of the level of polymorphism at the SSR loci. We demonstrate current limitations of the HRM method for the analysis of SSR loci.

Key words: Simple sequence repeat; High-resolution melting; Genotyping; Internal oligonucleotide calibrators; Limitations

\section{INTRODUCTION}

Gansu alpine fine wool sheep were originally bred in the Huangcheng District of Gansu Province, China, by crossbreeding Mongolian or Tibetan sheep with Xinjiang fine wool sheep and crossing the offspring with some fine wool breeds from the Union of Soviet Socialist Republics (USSR), such as Caucasian and Salsk sheep. The breed was approved by the Gansu provincial government in 1980 (Cheng, 1984). Since then, the breed was hybridized with Australian merino, New Zealand merino, and German mutton merino to improve the meat and wool quality. Today, the modern Gansu alpine fine wool sheep can be classified into 3 strains according to the fineness of wool, fine $(19.0 \mu \mathrm{m}<$ fiber diameter $<21.0 \mu \mathrm{m})$, superfine (fiber diameter $<19.0 \mu \mathrm{m}$ ), and dual-purpose strains (fiber diameter $>21.0 \mu \mathrm{m}$ ). Early research in our lab showed that Gansu alpine fine wool sheep had high genetic heterozygosity $\left(H_{\mathrm{E}}=0.85\right)$ and rich genetic diversity (Luo et al., 2009).

Microsatellites or simple sequence repeats (SSRs) are short DNA sequences in which a 1-6-bp motif is tandemly repeated (Wan et al., 2004). Because of their codominance, multiallelic nature, high reproducibility, extensive genome coverage, and amenability to automation and high-throughput genotyping (Agarwal et al., 2008; Kalia et al., 2011), they have been applied in many fields, such as genetic diversity assessment, phylogenetic studies, genome mapping, and population structure analysis (Zhang et al., 2007; d'Angelo et al., 2009; Zhong et al., 2010; Arora et al., 2011). However, an important bottleneck of SSR analysis is that the traditional protocols involve laborious polyacrylamide gels and carcinogenic DNA stains. Although capillary electrophoresis (CE) resolves these issues, the procedure requires postpolymerase chain reaction (PCR) handling and is costly.

High-resolution melting (HRM) is a homogenous, closed-tube method to analyze PCR products (Wittwer et al., 2003). It is based on changes in fluorescence of double-stranded (ds)DNA as a melting curve is performed because the intercalating fluorescent dye only binds dsDNA (Reed et al., 2007). PCR products are analyzed without gels and hazardous chemicals, such as ethidium bromide. Moreover, the melting process is faster, data analysis can be performed automatically in a few minutes, and the sensitivity is very high relative to other methods (Vossen et al., 2009). HRM has been widely used to detect single nucleotide polymorphisms (SNPs) and to scan mutations (Vorkas et al., 2010; Trujillo et al., 2012). Recently, more and more studies have focused on the genotyping of microsatellites by HRM. For example, Bosmali et al. (2012) applied the Bar-HRM method to 10 varieties of lentils to characterize the DNA barcoding region rpoC1 and finally identified alterations; Costa et al. (2010) genotyped Candida albicans isolates by combining fragment analysis, HRM analysis, and $\mathrm{SNaP}$ shot 
mini sequencing (2010); and Distefano et al. (2012) showed that 16 SSR markers produced distinct polymorphic melting curves among the Citrus spp that were investigated by HRM analysis. Among the 16 SSR markers that were investigated in Citrus spp, 10 showed more genotypes by HRM analysis than by CE because of the presence of SNPs in the amplicons. For the SSR markers without SNPs present in the flanking region, HRM also gave distinct melting curves that detected the same genotypes that were shown by CE analysis (Distefano et al., 2012). Yu et al. (2011) successfully used this method to identify genotypes of the $F_{2}$ generation and to detect SSR polymorphisms in different maize inbred lines. However, as far as we know, there have been using HRM for SSR genotyping in the ruminant sciences field. The main objective of our current experiment was to test whether HRM could be efficiently applied to genotype SSRs from sheep.

\section{MATERIAL AND METHODS}

\section{Sheep genomic DNA}

Twenty-four unrelated individual genomic DNA samples were obtained from the Gansu alpine fine wool sheep breed. The DNA was extracted from whole blood using the TIANGEN Genomic DNA Purification Kit (TIANGEN, Beijing, China). After extraction, samples were diluted to a final concentration of $20 \mathrm{ng} / \mu \mathrm{L}$ and then stored at $-20^{\circ} \mathrm{C}$ for subsequent analysis.

\section{Primer design}

Four microsatellite markers (Table 1) were used in this study, and all of them were from the panel recommended by ISAG/FAO for sheep diversity studies (CGRFA/WG-AnGR-6/10/Inf.7).

\begin{tabular}{|c|c|c|c|c|c|c|c|}
\hline \multirow[t]{2}{*}{ Marker } & \multirow[t]{2}{*}{ Accession No. } & \multirow[t]{2}{*}{ Sequence } & \multirow{2}{*}{$\begin{array}{l}\text { Allele size } \\
\text { range (bp) }\end{array}$} & \multicolumn{2}{|c|}{ Genotype No. } & \multicolumn{2}{|c|}{ Allele No. } \\
\hline & & & & $\mathrm{CE}$ & HRM & $\mathrm{CE}$ & HRM \\
\hline MAF209 & M80385 & $\begin{array}{l}\text { F-GATCACAAAAAGTTGGATACAACCGTGG } \\
\text { R-TCATGCACTTAAGTATGTAGGATGCTG }\end{array}$ & $98-136$ & 9 & 5 & 4 & 4 \\
\hline MCM140 & L38979 & $\begin{array}{l}\text { F-GTTCGTACTTCTGGGTACTGGTCTC } \\
\text { R-GTCCATGGATTTGCAGAGTCAG }\end{array}$ & $167-193$ & 12 & 4 & 7 & 7 \\
\hline CB226 & L20006 & $\begin{array}{l}\text { F-CTATATGTTGCCTTTCCCTTCCTGC } \\
\text { R-GTGAGTCCCATAGAGCATAAGCTC }\end{array}$ & $119-153$ & 19 & 8 & 10 & 10 \\
\hline SRCRSP5 & L22197 & $\begin{array}{l}\text { F-GGACTCTACCAACTGAGCTACAAG } \\
\text { R-GTTTCTTTGAAATGAAGCTAAAGCAATGC }\end{array}$ & $126-158$ & 7 & 4 & 4 & 4 \\
\hline Mean & & & & 11.75 & 5.25 & 6.25 & 6.25 \\
\hline
\end{tabular}

$\mathrm{F}=$ forward primer; $\mathrm{R}=$ reverse primer.

\section{PCR amplifications}

For CE, PCRs included $0.3 \mu \mathrm{M} 2$ specific labeled FAM primers, approximately 30 ng template DNA, $4.0 \mathrm{mM}$ dNTPs, 10X PCR buffer, and 1 U Premix Taq DNA polymerase (TaKaRa, Dalian, China). PCR was performed at $95^{\circ} \mathrm{C}$ for $4 \mathrm{~min} ; 30$ cycles of $95^{\circ} \mathrm{C}$ for $30 \mathrm{~s}$, 
$58^{\circ} \mathrm{C}$ for $30 \mathrm{~s}$, and $72^{\circ} \mathrm{C}$ for $30 \mathrm{~s}$; and a final cycle of $72^{\circ} \mathrm{C}$ for $10 \mathrm{~min}$ on a Bio-Rad thermo cycler (California, USA). For HRM analysis, PCR amplifications were performed in a total volume of $10 \mu \mathrm{L}$ on a Bio-Rad thermocycler, which contained $2 \mu \mathrm{L}$ genomic DNA ( $20 \mathrm{ng} / \mu \mathrm{L})$, $5 \mu \mathrm{L} 2 \mathrm{X}$ Taq PCR Master Mix (Tiangen Biotech Co., Ltd., Beijing, China), $1 \mu \mathrm{L}$ LC Green (Idaho Technology, Alameda, CA, USA), $0.05 \mu \mathrm{M}$ internal oligo nucleotide calibrators, and $0.25 \mu \mathrm{M}$ primers. The PCR protocol was as follows: denaturation at $94^{\circ} \mathrm{C}$ for $2 \mathrm{~min} ; 35$ cycles of denaturation at $94^{\circ} \mathrm{C}$ for $30 \mathrm{~s}$, annealing at $60^{\circ} \mathrm{C}$ for $25 \mathrm{~s}$, and extension at $72^{\circ} \mathrm{C}$ for $25 \mathrm{~s}$; and extension at $72^{\circ} \mathrm{C}$ for $7 \mathrm{~min}$. Calibrators were used as recommended (Gundry et al., 2008). After completing amplification, a final denaturation and reannealing protocol was performed by raising the temperature to $94^{\circ} \mathrm{C}$ for $30 \mathrm{~s}$ followed by a $28^{\circ} \mathrm{C}$ hold for $30 \mathrm{~s}$.

\section{HRM using the LightScanner ${ }^{\circledR}$ platform and data analysis}

PCR products were detected according to the method by Hofinger et al. (2009). The 96-well plate was centrifuged and used directly for HRM using the LightScanner ${ }^{\mathbb{B}}$ instrument. Data were collected from $50^{\circ}-96^{\circ} \mathrm{C}$ at a ramp rate of $0.1^{\circ} \mathrm{C} / \mathrm{s}$. Melting profiles were calibrated by first using a smoothing spline to approximate the fluorescence data. Then, the melting temperature $(\mathrm{Tm})$ values were computed for each internal oligonucleotide calibrator that was present using the interpolated apex of the derivative spline data. Shift and linear scale factors were applied to align each calibrator. This shifting process aligns the amplicon melting profiles and minimizes variation. To ensure data quality, PCR amplification was analyzed by assessing the fluorescence value, which reflects the amplification efficiency. The data from low-quality amplification and drifting curves were removed from HRM analysis. The data obtained were analyzed using the LightScanner ${ }^{\circledR}$ analytical software with Call-IT ${ }^{\mathrm{TM}} 2.0$ (Idaho Technology). The normalization and the temperature shifting of the melting curves were finished according to the LightScanner ${ }^{\circledR}$ Operator's Manual (Idaho Technology).

\section{CE analysis}

PCR product $(0.5 \mu \mathrm{L})$ was mixed with $9 \mu \mathrm{L}$ formamide and $0.5 \mu \mathrm{L}$ LIZ-500 size standard and was denatured at $95^{\circ} \mathrm{C}$ for 4 min before separation in the ABI 3730XL (Applied BioSystems, Foster, CA, USA). Analysis was conducted using the Gene marker 1.7 software.

\section{RESULTS}

Four primer pairs amplifying fragments that were less than $200 \mathrm{bp}$ produced clear genotyping profiles that were generated from both HRM and CE analysis (Table 1). All 4 of the SSR markers showed polymorphic melting curves by HRM analysis. In total, 25 alleles and 21 genotypes were detected with the average of 6.25 alleles per locus. In addition, there was an average of 5.25 genotypes in the population under investigation. For CE, we also detected 25 alleles for the same samples; however, 46 genotypes were investigated in the population with an average of 11.25 genotypes per locus (Table 1 ).

For the 4 primers, every primer showed more genotypes by CE analysis than HRM. For example, 8 genotypes of MAF209 were detected by CE (Figure 1), but only 5 genotypes 
were detected by HRM (Figure 2). Except for amplicons 104/115 (orange curve) and 115/125 (green curve), which were genotyped well, the others were misclassified. Although HRM failed to detect different genotypes, those amplicons contained the same alleles, in which all of the green curves were allele 104, the red curves were allele 120, and gray curves were allele 115.

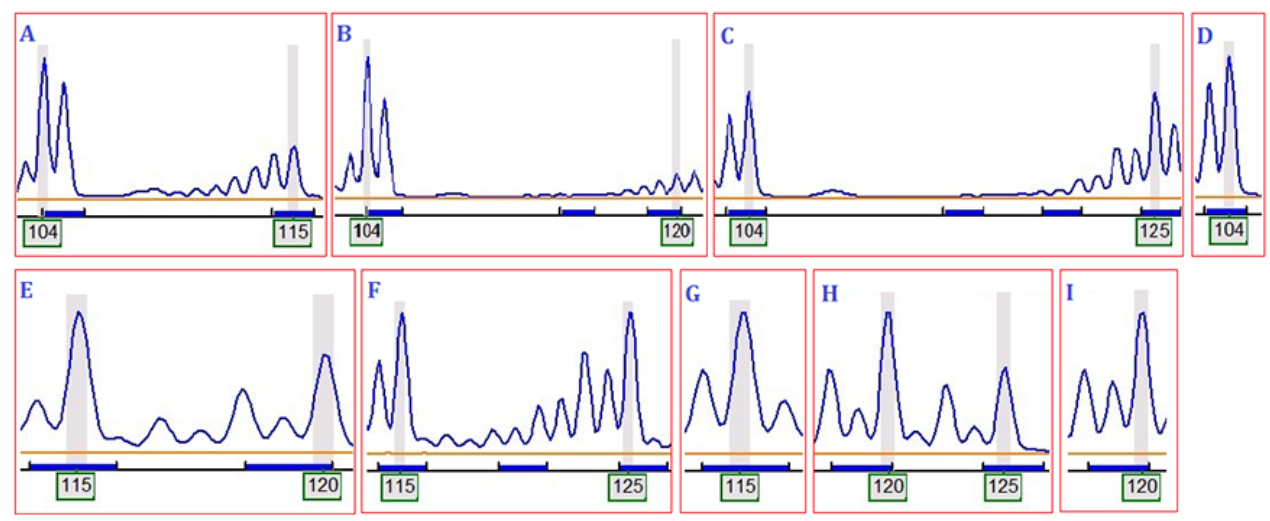

Figure 1. Capillary electrophoresis (CE) profiles of the genotypes of the MAF209 marker. CE profiles of 9 different genotypes. Four different alleles $(104,115,120$, and 125 bp) from 3 homozygous and 6 heterozygous genotypes.

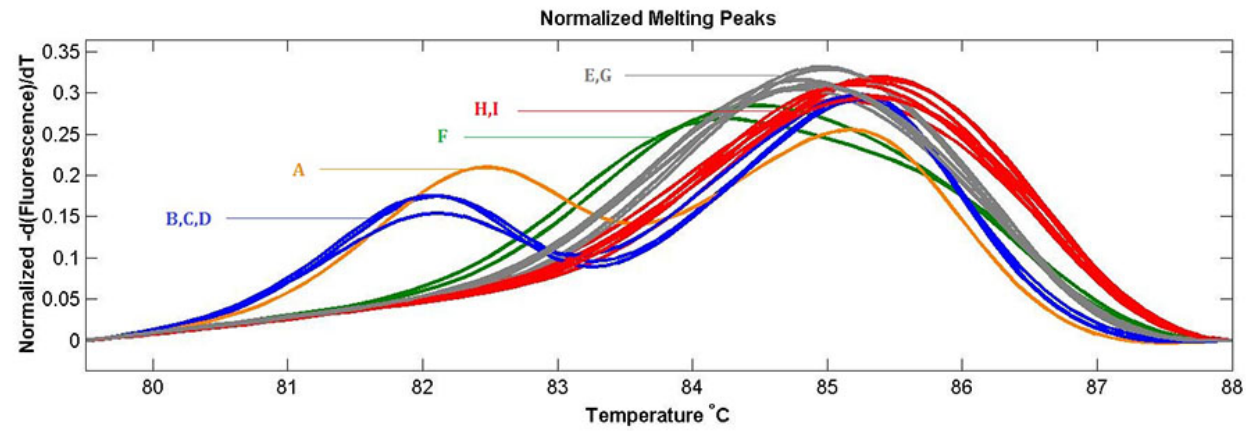

Figure 2. Normalized high-resolution melting (HRM) peaks of the MAF209 marker. Five genotypes are shown: 2 genotypes were accurately distinguished (the orange curve included the 104 and 115 alleles, and the green curve included the 115 and 125 alleles); the gray curve included 2 genotypes, both of which consisted of the 115 allele; the red curve included the 120 allele; and the blue curve included the 104 allele.

For MCM140, four genotypes were detected by HRM, and 12 genotypes were identified by $\mathrm{CE}$. Moreover, it was difficult to detect the genotypes by the normalized melting curves (Figures 3 and 4).

Both of the methods discovered 10 alleles for CB226, but there were 19 genotypes by $\mathrm{CE}$, and only 8 genotypes by HRM. Indeed, the polymorphism of the loci is so high that it is very difficult to genotype the loci using HRM. The SRCRSP5 locus, which contains 4 alleles, had 7 genotypes by CE and 4 genotypes by HRM. 

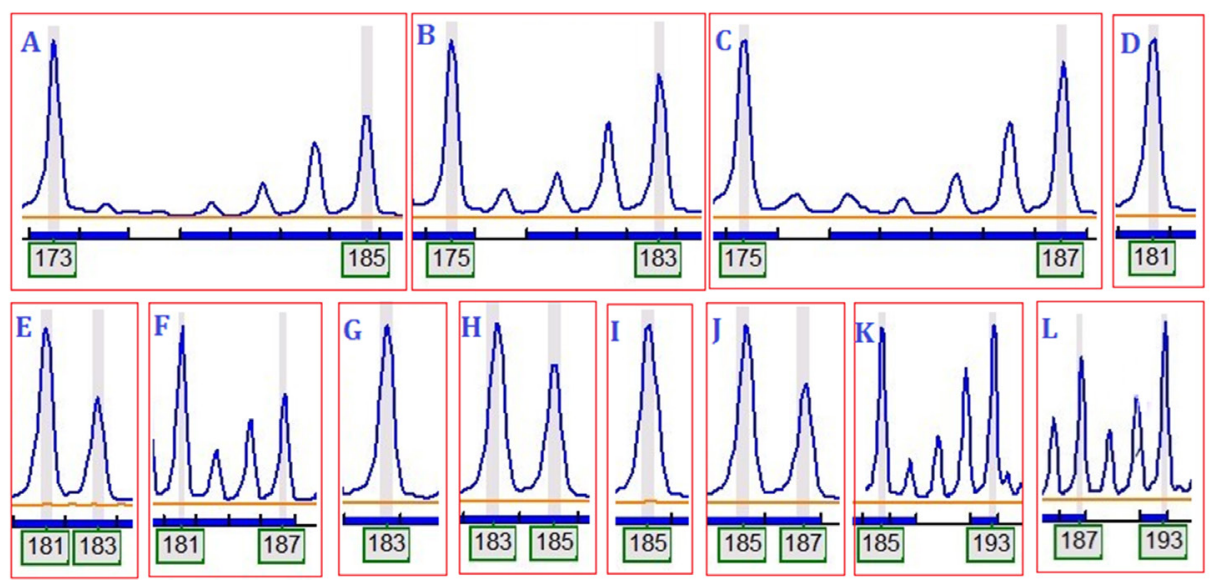

Figure 3. Capillary electrophoresis (CE) profiles of the genotypes analyzed for the MCM140 marker. The CE profiles of 12 different genotypes are shown. Seven different alleles $(173,175,181,183,185$, 187, and $193 \mathrm{bp})$ were detected from 3 homozygous and 9 heterozygous genotypes.

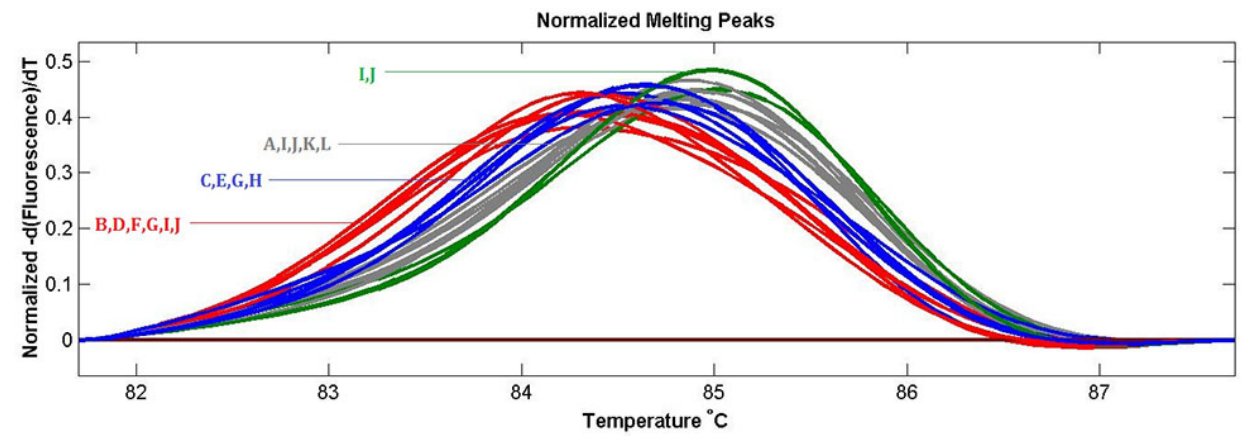

Figure 4. Normalized HRM peaks of the MCM140 marker, which showed 4 genotypes. Genotypes I (185/185) and $\mathrm{J}(185 / 187)$ appeared simultaneously in different colors (the green, gray, and red curves).

\section{DISCUSSION}

Because this approach could be limited by physical and chemical components of the system that contribute to intersample melting variation, some homozygotes display similar melting curves and Tm. Internal oligonucleotide calibrators can improve genotyping by melting curve analysis (Gundry et al., 2008). Therefore, we applied them in our experiment. As far as we know, HRM analysis is an effective approach to genotype using SSR markers at the interspecific level, but it is not effective at the intraspecific level because the similarity is still very high for those samples (Bosmali et al., 2012; Distefano et al., 2012). We applied this approach to genotype the microsatellite loci of sheep.

The purpose of this study was not a direct comparison of the CE and HRM methods; rather, this study assessed the capability of the HRM method to genotype SSRs for polymorphisms in animals. Therefore, we aimed to determine potential advantages and/or limitations 
of the HRM method for laboratories planning to adopt this new technique. Many studies have reported the high sensitivity, ease of data interpretation, and the low cost associated with the use of CE for SSR analysis (Costa et al., 2010; Yu et al., 2011; Bosmali et al., 2012; Distefano et al., 2012). Depending on the fluorescence primers used for amplification, the cost of reagents to perform CE screening is half to three-quarters that required for HRM using these platforms and dyes. Ignoring these advantages, $\mathrm{CE}$ and other gel-based methods are gradually and unavoidably being replaced by more high-throughput methods such as HRM curve analysis, which requires less technical expertise and hands-on time and excludes hazardous substances, such as polyacrylamide, formamide, and TEMED, that are used in CE. These new methods, however, require extensive and comprehensive validation before replacing the current techniques.

HRM has proven to be an efficient and labor-saving way to detect sequence variations such as SNPs in humans, plants, and microorganisms (Millat et al., 2009; Derzelle et al., 2011; Li et al., 2012). In plants, such an approach has been applied to detect SSRs, which was used for genotype identification and authenticity testing (Jaakola et al., 2010; Ganopoulos et al., 2011; Distefano et al., 2012; Madesis et al., 2012). Moreover, it can also be used for mutation scanning (Vorkas et al., 2010). The application of HRM to discriminate SSR genotypes in domestic animals has not been reported. In this study, we analyzed 4 SSR markers of sheep using HRM, and the results were compared with those produced from CE analysis. In theory, physical and chemical components of the system that contribute to inter sample melting variation would be observably decreased as a result of using internal oligonucleotide calibrators. However, the results were different than expected.

The first possible cause is that, the high level of polymorphisms of microsatellite loci could explain the differences. In plants, HRM analysis is an effective approach to genotype using SSR markers at the inter specific level, but it is not effective at the intra specific level (Distefano et al., 2012) because similarity is still very high for those samples. This has been considered the main reason why the molecular marker systems that have been used so far are not effective in discriminating genotypes of such origin at the intraspecific level. When the number of alleles is low, the analysis gives few curves, and the delineation of the genotypes is easy (Mader et al., 2008). When the number of alleles increases to 7, the delineation of the curves becomes more difficult and requires artificial mixtures of samples for comparison. The research about the genotyping of yeast denied this idea, and HRM analysis did not allow the discrimination of the SSR genotypes when 11 genotypes were detected by CE despite adjusting multiple parameters (Costa et al., 2010).

In our experiment, many SSR marker genotypes were detected (Table 1). Among them, the marker CB226 was investigated, which had 10 alleles and 19 genotypes and was difficult to genotype. Although 4 alleles and 8 genotypes were detected for marker MAF209, this is still not an accurate method to genotype. This may be caused by the complexity and high variability of the loci that were analyzed in Gansu alpine fine wool sheep.

Second, the size of the small amplicons could explain the differences between the expected and observed results. Although it was reported that the size range of many amplicons that were genotyped for SSRs were greater than $200 \mathrm{bp}$ and were genotyped very well (Distefano et al., 2012), HRM of small amplicons (40-90 bp) improves homozygote detection sensitivity because Tm differences are greater than in larger amplicons (Gundry et al., 2008).

Thirdly, high-resolution DNA melting instruments can account for the differences between observed and expected results. Almost all of the reports of SSR genotyping have used a Rotor-gene 6000 or 6500 instrument rather than a Light Scanner. The apparent temperature variation within a genotype was highly dependent on the instrument used (Herrmann et al., 2007). 
Our assessment of this technology for SSR genotyping revealed important limitations. This was not only apparent for large numbers of alleles but also introduced the potential of either missing or misclassifying genotypes in cases of a small number of alleles. Animals have far fewer SSR loci than plants, so we could not select enough effective loci that had a low level of polymorphism like other species. However, we believe that these limitations will be resolved as the technology is further developed in the near future.

\section{ACKNOWLEDGMENTS}

Research supported by the Central Level, Scientific Research Institutes for Basic R\&D Special Fund Business (\#BRF100102), the Earmarked Fund for Modern China Wool \& Cashmere Technology Research System (\#nycytx-40-3), and the National High Technology Research and Development Program of China ("863" Program) (\#2008AA101011-2).

\section{REFERENCES}

Agarwal M, Shrivastava N and Padh H (2008). Advances in molecular marker techniques and their applications in plant sciences. Plant Cell Rep. 27: 617-631.

Arora R, Bhatia S, Mishra BP and Joshi BK (2011). Population structure in Indian sheep ascertained using microsatellite information. Anim. Genet. 42: 242-250.

Bosmali I, Ganopoulos I, Madesis P and Tsaftaris A (2012). Microsatellite and DNA-barcode regions typing combined with High Resolution Melting (HRM) analysis for food forensic uses: A case study on lentils (Lens culinaris). Food Res. Int. 46: 141-147.

Cheng PL (1984). Sheep breeds of China. World Anim. Rev. 49: 19-24.

Costa JM, Garcia-Hermoso D, Olivi M, Cabaret O, et al. (2010). Genotyping of Candida albicans using length fragment and high-resolution melting analyses together with minisequencing of a polymorphic microsatellite locus. $J$. Microbiol. Methods 80: 306-309.

d'Angelo F, Albenzio M, Sevi A, Ciampolini R, et al. (2009). Genetic variability of the Gentile di Puglia sheep breed based on microsatellite polymorphism. J. Anim. Sci. 87: 1205-1209.

Derzelle S, Mendy C, Laroche S and Madani N (2011). Use of high-resolution melting and melting temperature-shift assays for specific detection and identification of Bacillus anthracis based on single nucleotide discrimination. $J$. Microbiol. Methods 87: 195-201.

Distefano G, Caruso M, La Malfa S, Gentile A, et al. (2012). High resolution melting analysis is a more sensitive and effective alternative to gel-based platforms in analysis of SSR - an example in citrus. PLoS One 7: e44202.

Ganopoulos I, Argiriou A and Tsaftaris A (2011). Microsatellite high resolution melting (SSR-HRM) analysis for authenticity testing of protected designation of origin (PDO) sweet cherry products. Food Control 22: 532-541.

Gundry CN, Dobrowolski SF, Martin YR, Robbins TC, et al. (2008). Base-pair neutral homozygotes can be discriminated by calibrated high-resolution melting of small amplicons. Nucleic Acids Res. 36: 3401-3408.

Herrmann MG, Durtschi JD, Wittwer CT and Voelkerding KV (2007). Expanded instrument comparison of amplicon DNA melting analysis for mutation scanning and genotyping. Clin. Chem. 53: 1544-1548.

Hofinger BJ, Jing HC, Hammond-Kosack KE and Kanyuka K (2009). High-resolution melting analysis of cDNA-derived PCR amplicons for rapid and cost-effective identification of novel alleles in barley. Theor. Appl. Genet. 119: 851-865.

Jaakola L, Suokas M and Häggman H (2010). Novel approaches based on DNA barcoding and high-resolution melting of amplicons for authenticity analyses of berry species. Food Chem. 123: 494-500.

Kalia RK, Rai MK, Kalia S, Singh R, et al. (2011). Microsatellite markers: an overview of the recent progress in plants. Euphytica 177: 309-334.

Li XH, Acharya A, Farmer AD, Crow JA, et al. (2012). Prevalence of single nucleotide polymorphism among 27 diverse alfalfa genotypes as assessed by transcriptome sequencing. BMC Genomics 13: 568.

Luo LT, Yang BH, Shi ZG and Lang X (2009). Analysis on the polymorphism of fifteen microsatellite loci in high quality fine wool strain of Gansu alpine fine wool sheep. China Herbivores 29: 8-11.

Mader E, Lukas B and Novak J (2008). A strategy to setup codominant microsatellite analysis for high-resolution-meltingcurve-analysis (HRM). BMC Genet. 9: 69. 
Madesis P, Ganopoulos I, Anagnostis A and Tsaftaris A (2012). The application of Bar-HRM (Barcode DNA-High Resolution Melting) analysis for authenticity testing and quantitative detection of bean crops (Leguminosae) without prior DNA purification. Food Control 25: 576-582.

Millat G, Chanavat V, Rodriguez-Lafrasse C and Rousson R (2009). Rapid, sensitive and inexpensive detection of SCN5A genetic variations by high resolution melting analysis. Clin. Biochem. 42: 491-499.

Reed GH, Kent JO and Wittwer CT (2007). High-resolution DNA melting analysis for simple and efficient molecular diagnostics. Pharmacogenomics 8: 597-608.

Trujillo AI, Peñagaricano F, Grignola MP and Nicolini P (2012). Using high resolution melting analysis to identify variation of NPY, LEP and IGF-1 genes in Angus cattle. Livest. Sci. 146: 193-198.

Vorkas PA, Christopoulos K, Kroupis C and Lianidou ES (2010). Mutation scanning of exon 20 of the BRCA1 gene by high-resolution melting curve analysis. Clin. Biochem. 43: 178-185.

Vossen RH, Aten E, Roos A and den Dunnen JT (2009). High-resolution melting analysis (HRMA): more than just sequence variant screening. Hum. Mutat. 30: 860-866.

Wan QH, Wu H, Fujihara T and Fang SG (2004). Which genetic marker for which conservation genetics issue? Electrophoresis 25: 2165-2176.

Wittwer CT, Reed GH, Gundry CN, Vandersteen JG, et al. (2003). High-resolution genotyping by amplicon melting analysis using LCGreen. Clin. Chem. 49: 853-860.

Yu RH, Shan XH, Wang S, Li XH, et al. (2011). A screening method for detecting simple sequence repeat (SSR) polymorphism of Zea mays using high-resolution melting-curve analysis. Afr. J. Biotechnol. 10: 16443-16447.

Zhang JH, Xiong YZ, Zuo B and Lei MG (2007). Genetic analysis and linkage mapping in a resource pig population using microsatellite markers. J. Genet. Genomics 34: 10-16.

Zhong T, Han JL, Guo J, Zhao QJ, et al. (2010). Genetic diversity of Chinese indigenous sheep breeds inferred from microsatellite markers. Small Ruminant Res. 90: 88-94. 